\title{
PERCEPCIÓN SOBRE EL CONSUMO DE SUSTANCIAS PSICOACTIVAS POR ESTUDIANTES DE MEDICINA DE LA PROVINCIA DE ICA, PERÚ 2010
}

\author{
Adán Antonio Pineda-Rodríguez ${ }^{1, a}$ \\ 1. Facultad de Medicina. Universidad Nacional San Luis Gonzaga. Ica. Perú. \\ a. Interno de medicina
}

\section{RESUMEN}

Con el objetivo de determinar la percepción sobre el consumo de sustancias psicoactivas en estudiantes de la provincia de Ica se realizo un estudio de tipo descriptivo transversal, para el cual se aplicó una encuesta estructurada a una muestra no probabilística estratificada, para todos los años de estudios, en la Facultad de Medicina de la Universidad San Luis Gonzaga de Ica. Para el análisis de los datos se usó estadística descriptiva en el software Excel 2007. El número de encuestados fue de 195, con una edad promedio de $22 \pm 3$ años. La percepción: peligro o muy peligroso, sobre el consumo de sustancias psicoactivas, fue del 78,5\% para fumar cigarrillos, $83,6 \%$ para el consumo de bebidas alcohólicas frecuentemente; un $80 \%$ considera nada peligroso o poco peligroso al consumo de café. Concluimos que existe una alta percepción de peligro sobre el consumo de sustancias psicoactivas; sin embargo, un gran porcentaje de estos estudiantes, ha consumido alguna vez un tipo de ellas. (Rev. méd. panacea 2011; 1:44-46).

Palabras claves: sustancias psicoactivas, consumo, estudiantes de medicina.

\section{PERCEPTION ABOUT INTAKE OF PHYCHOACTIVE SUBSTANCES BY MEDICAL STUDENTS FROM ICA PROVINCE, PERU 2010}

\begin{abstract}
The objective is to determine the perception about intake of psychoactive substances used among medical students in the province of Ica. A descriptive- transversal study was realized, for which a structured survey was applied to a non-probabilistic stratified sample, for all years of study at the Faculty of Medicine of the San Luis Gonzaga de Ica University. For data analysis, descriptive statistics were used SPSS v.17 software and Excel 2007 software. The number of respondents was 195, with an average age of $22 \pm 3$ years. Perception: Dangerous or Very dangerous, for psychoactive substances intake, was $78.50 \%$ for smoking, $83.60 \%$ for the use of alcoholic beverages frequently. $80 \%$ consider non-dangerous or little dangerous coffee consumption. Conclusions: There is a high perception of danger about intake of psychoactive substances, but a large percentage of these students had ever used one type of these. (Rev. méd. panacea 2011; 1:44-46).
\end{abstract}

Key words: psychoactive substance, medical students.

\section{INTRODUCCIÓN}

Los psicoactivos, se definen como cualquier sustancia natural o sintética que altere de alguna forma el funcionamiento del sistema nervioso central ${ }^{(1)}$.

En Perú, el Centro de Información y Educación para la Prevención del Consumo de Drogas (CEDRO), reporta que aproximadamente $82,10 \%$ de estudiantes de medicina, habían fumado alguna vez en la vida y que la droga ilegal más consumida entre universitarios es la Marihuana (23\%), seguida por el Clorhidrato de Cocaína $(4,80 \%)^{(2)}$.

Sin embargo, entre los universitarios, lo más frecuente es el empleo de "drogas sociales", observándose que el consumo alguna vez en la vida de bebidas alcohólicas alcanza el $97,70 \%$ y, el de tabaco es del $77,50 \%{ }^{(3)}$.
El objetivo de nuestra investigación fue determinar la percepción sobre el consumo de sustancias psicoactivas en estudiantes, de medicina de la provincia de Ica durante el primer semestre del 2010.

\section{MATERIAL Y MÉTODOS}

Se realizo un estudio de tipo transversal y descriptivo.

La población en estudio estuvo constituida por 502 estudiantes registrados durante el primer semestre del 2010, de primero a sexto año de la Facultad de Medicina "Daniel Alcides Carrión" de la Universidad Nacional San Luis Gonzaga de Ica. 
La muestra con la que se realizó el estudio estuvo constituida por 195 estudiantes, elegidos por conveniencia, considerando muestras homogéneas por año de estudio.

La recolección de datos se llevó a cabo en los salones de clases, coordinando previamente con el docente de la asignatura en curso. La aplicación de las encuestas fue ejecutada por seis alumnos de la Facultad de Medicina "Daniel A. Carrión", los cuales fueron previamente capacitados; estos invitaron a los estudiantes a participar del estudio y firmar el consentimiento informado, garantizando la intimidad y anonimato.

Para el estudio se estructuró un cuestionario tomando como base el Estandarizado del "Sistema Interamericano de Datos Uniformes Sobre Consumo de Drogas (SIDUC)", brindado por el Observatorio Interamericano sobre Drogas (OID)", rama de estadística, de información e investigación científica de la Comisión Interamericana para el Control del Abuso de Drogas (CICAD). La validación del cuestionario se realizo mediante la opinión de expertos, en número de dos. La confiabilidad del mismo se obtuvo mediante el Testretest. Para la tabulación y el análisis de datos se utilizó el programa Microsoft office Excel 2007.

\section{RESULTADOS}

El número de encuestados fue 195, con un promedio de edad de 22 \pm 3 años (Mínimo 17, máximo 38 años), de los cuales el $48 \%$ fueron hombres. La distribución por año de estudio fue homogénea.

La edad promedio para el inicio del consumo de tabaco fue de 16.0 \pm 2,6 años; para el inicio de consumo de alcohol fue de 16,6 $\pm 2,4$ años, siendo la edad mínima de 7 y la máxima de 25 años. Para el consumo de tranquilizantes la edad

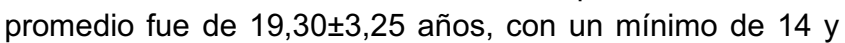
máximo de 26 años; a diferencia de la edad promedio para el consumo de estimulantes que fue de 17,27 $\pm 3,57$ años, con un mínimo y un máximo 24 años.

La percepción: Peligro o Muy Peligroso, sobre el consumo de sustancias psicoactivas, fue del: $78,5 \%$ para fumar, $83,6 \%$ para el uso frecuente de bebidas alcohólicas, $81,5 \%$ para el uso frecuente de tranquilizantes y/o estimulantes; $88,2 \%$ para el uso de Diazepam y/o Valium, 92,3\% para el uso frecuente de solventes, 95,4\% para el uso frecuente de Marihuana. Un $80 \%$ considera nada peligroso o poco peligroso al consumo de café alguna vez, 66,2\% para el uso algunas veces de bebidas energizantes.

A la pregunta, si tienen amigos que consumen alcohol en forma exagerada, un $16,92 \%$ respondió que tenía al menos un amigo y un $38,97 \%$ que tenían varios amigos. El $10,26 \%$ refirió tener un amigo que consumía drogas ilícitas. Cuando se pregunto, sobre la dificultad para conseguir drogas ilícitas las respuestas fueron: 19,49\% para Muy difícil/difícil, $67,18 \%$ para Indiferente/ No sé, y $12,82 \%$ para Fácil/muy fácil. $\mathrm{Si}$, tuvieran la ocasión de probar una droga ilícita, $2,05 \%$ de universitarios encuestados lo haría.

El $35,38 \%$ ha recibido cursos de prevención sobre el consumo de psicoactivos. Un $77,44 \%$ refirió sentirse bien informado sobre el consumo de psicoactivos. El 76,92\% de encuestados manifestó estar en Total desacuerdo/Desacuerdo con la legalización del consumo de drogas en el Perú.

\section{DISCUSIÓN}

Nuestros resultados muestran que la edad de inicio para el consumo de sustancias psicoactivas es más frecuente en menores de edad, siendo este resultado similar a lo encontrado en un estudio realizado por CEDRO ${ }^{(3)}$.

Se observa además que el promedio de edad del inicio del consumo de alcohol es de 16,6 \pm 2 años, lo cual refleja la corta edad de inicio del consumo. Este resultado es similar al obtenido en un trabajo realizado por López y col. donde la edad media del primer contacto con el consumo de alcohol fue a los quince años ${ }^{(4)}$.

En cuanto a la percepción del riesgo sobre diversas sustancias psicoactivas, se pudo observar que la sustancia considerada, por los universitarios, como muy peligrosa o peligrosa fue fumar frecuentemente marihuana $(90,4 \%)$. Este resultado difiere con un trabajo hecho en Argentina, donde obtuvieron como respuesta una menor percepción de riesgo para el consumo de esta sustancia psicoactiva $(69,6 \%)^{(5)}$.

El consumo de tabaco es considerado por los universitarios como Nada peligroso o Poco peligroso en un $21,50 \%$, esto es mayor al $10,97 \%$ de universitarios argentinos que consumen tabaco ${ }^{(5)}$.

Al considerar la categoría: No sabe o indiferente, se encuentra que un $10,8 \%$ es para consumir Diazepam/Valium algunas veces; de igual manera, para consumir tranquilizantes alguna vez. Nuestros resultados eran superiores que los hallados en un trabajo realizado en Argentina, donde uno de cada diez estudiantes desconocía que tan peligroso es el consumo de clorhidratos de cocaína, alucinógenos, solventes e inhalables ${ }^{(5)}$.

Se observó también que un $76,92 \%$ de encuestados está en desacuerdo con legalizar el consumo de drogas en el Perú, este resultado concuerda con lo manifestado por 
CEDRO el año 2008 , donde un $77,7 \%$ opinaba que no se debe legalizar el consumo de drogas ${ }^{(6)}$.

Estudios en Estados Unidos, encontraron que $17 \%$ de los hombres y $11 \%$ de las mujeres universitarias refirieron usar estimulantes sin prescripción médica.

En Honduras, Buchanan \& Pillon (2008) ${ }^{(2)}$, reportaron que el $25 \%$ de la población de universitarios usan algún tipo de droga $^{(8)}$.

El presente informe, es considerado como basal, el primero de nuestra investigación. Más adelante se piensa realizar un análisis inferencial para determinar las motivos que podrían estar orientados hacia FAVORECER el consumo de sustancias psicoactivas.

Por otro lado, considero que se deben implementar charlas educativas y motivadoras, ya que un alto porcentaje de estudiantes universitarios manifiesta que el uso de tranquilizantes no es peligroso; esto podría llevarlos al consumo de psicoactivos, convirtiéndose en parte de la gran población consumidora.

\section{REFERENCIAS BIBLIOGRÁFICAS}

1. Organización Mundial de la Salud. Neurociencia del consumo y dependencia de sustancias psicoactivas. Ginebra: OMS. 2004 Disponible en: http://www.who.int/substance_abuse/publications/en/Ne uroscience.pdf (accesado 12 de octubre 2009).

2. Alarcón R, Perotti G, Zavala M. Factores de riesgo y protección para el consumo de sustancias psicoactivas en estudiantes universitarios. Consensus. 2004; 8(9): 9-18.

3. Centro de Información y Educación para la Prevención del Consumo de Drogas. CEDRO
Estudio sobre drogas en universitarios de lima.. Lima 2005. 13 pp. Disponible en: http://www.cedro.org.pe/ebooks/universitarios.pdf (accesado 09 octubre 2009).

4. López MJ, Santín C, Torrico E, Rodríguez J. Consumo de sustancias psicoactivas en una muestra de jóvenes universitarios. Rev Psicología y Salud. 2003; 13(1)

5. Secretaría de Programación para la Prevención de la Drogadicción y la Lucha contra el Narcotráfico. Consumo de sustancias psicoactivas en estudiantes universitarios. Argentina: Observatorio Argentino de Drogas. 2005. 5pp. Disponible en: http://www.cedro.org.pe/ebooks/universitarios.pdf (accesado 09 octubre 2009)

6. Centro de Información y Educación para la Prevención del Consumo de Drogas. Problemas de las drogas en el Perú. Perú: CEDRO. 2008 Disponible en: http://www.cedro.org.pe/ebooks/ebooks3.htm (accesado 09 octubre 2009).

7. Organización Panamericana de la Salud. Centro de Información y Educación para la Prevención del Abuso de Drogas. Encuesta Mundial de Profesionales de la Salud: El uso de tabaco en estudiantes de tercer año de Medicina, Enfermería y Farmacia - Perú 2006Lima: CEDRO, 2007. 50 p.

8. Buchanan JC, Pillon SC. Uso de drogas entre estudiantes de medicina, tegucigalpa, Honduras. Rev latino-americana de enfermagem. 2008; 16: 595-60

\section{Correspondencia}

Adán Pineda-Rodríguez

adan_pineda98@hotmail.com 